DOI: https://doi.org/10.46296/yc.v4i7.0052

\title{
RESILIENCIA Y ANSIEDAD EN PERSONAS CON COVID-19 EN MANTA, MANABÍ, ECUADOR
}

\section{RESILIENCE AND ANXIETY IN PEOPLE WITH COVID-19 IN MANTA, MANABÍ, ECUADOR}

\author{
Tirino-Quijije Daniel August ${ }^{1 *}$; Giniebra-Urra Ricardo² \\ 1,2 Universidad Técnica de Manabí, UTM. Portoviejo, Ecuador. \\ *Correo: dtirino0521@utm.edu.ec
}

\begin{abstract}
Resumen
La investigación determina los niveles de ansiedad y resiliencia, así como la relación entre estas categorías, en personas que fueron diagnosticadas con el virus de COVID-19 en la ciudad de Manta, provincia Manabí, la cual está dentro de las provincias con más casos de infección del Ecuador. Se estudia la relación existente entre el nivel de resiliencia y la ansiedad, asociados a la condición de estar atravesando por la enfermedad que produce el virus. La resiliencia es un factor protector para la ansiedad durante situaciones de estrés, de importancia para el bienestar integral de las personas. El estudio de estas categorías en las circunstancias descritas de la pandemia por coronavirus resulta pertinente, por cuanto aporta información valiosa acerca de los efectos psicológicos del COVID-19. La población está constituida por un grupo de personas que habitan en la ciudad de Manta, diagnosticadas con COVID-19 durante la pandemia de 2020, en los meses de junio y julio. Se realizó una entrevista abierta para determinar los niveles de expresión de las variables estudiadas. Se aplicaron las Pruebas de Ansiedad de Hamilton y de Resiliencia SV-RES. Como resultado se concluye, que existe una relación entre estas variables. Los resultados obtenidos indican que las personas mantienen un nivel de resiliencia como tendencia alto y la ansiedad se expresa en niveles bajos. En cuanto a la relación entre las mismas, se aprecia que los niveles de resiliencia encontrados permiten un mejor control de la ansiedad.
\end{abstract}

Palabras clave: Resiliencia, salud mental, pandemia.

\begin{abstract}
The following research tries to determine the levels of anxiety and resilience, as well as the relationship between these variables, in people who were diagnosed with the COVID-19 virus in the city of Manta, Manabí province, which is within the provinces with more cases of infection in Ecuador. The existing relationship between the level of resilience and anxiety is studied, associated with the condition of going through the disease caused by the virus. Resilience is a protective factor for anxiety during stressful situations, important for the overall well-being of people. The study of these categories in the described circumstances of the coronavirus pandemic is relevant, as it provides valuable information about the psychological effects of COVID-19. The population is made up of a group of people who live in the city of Manta, diagnosed with COVID-19 during the 2020 pandemic, between the months of June and July. An open interview was conducted to determine the levels of expression of the variables studied. The Hamilton Anxiety Test and the SV-RES Resilience Test were applied. As a result, it is concluded that there is a relationship between these variables. The results obtained indicate that people maintain a level of resilience as a high tendency and anxiety is expressed at low levels. Regarding the relationship between them, it is appreciated that the levels of resilience found allow a better control of anxiety.
\end{abstract}

Keywords: Resilience, mental health, pandemic.

Información del manuscrito:

Fecha de recepción: 11 de mayo de 2020.

Fecha de aceptación: 09 de julio de 2020.

Fecha de publicación: 10 de julio de 2020. 


\section{Introducción}

El COVID-19 ha tenido una clara influencia actualmente en el mundo, las experiencias y conductas que se tienen difieren de manera extensa, pero como factor común está la afectación mental que produce la existencia del virus. Probablemente represente una situación nueva, donde los seres humanos experimenten conductas asociadas a preocupaciones por su salud, viendo distante la posibilidad de regresar a una situación de normalidad como se vivía anteriormente.

El impacto de esta enfermedad ha producido un cambio significativo en los comportamientos habituales, hábitos, costumbres y modos de relación de los seres humanos, quienes se someten a nuevas exigencias para tratar de preservar su estado de salud ante la amenaza real existente.

Las reacciones humanas ante situaciones percibidas como amenazantes son estudiadas desde la Psicología. Algunas categorías como resiliencia y ansiedad pueden ser tomadas, para explorar aquellas respuestas de la persona ante estas situaciones. Las mismas dan cuenta de las posibilidades o recursos con que cuenta una persona para poder sortear la adversidad.

Es por ello por lo que este estudio intenta conocer cómo se manifiestan los niveles resiliencia y ansiedad de personas que han tenido el COVID19 y la relación que se da entre estas cualidades psicológicas.

El COVID-19, según la ONU (2020) se define como:

“(...) una amplia familia de virus que pueden causar diversas afecciones, desde el resfriado común hasta enfermedades más graves (...) Un nuevo coronavirus es una nueva cepa de coronavirus que no se había encontrado antes en el ser humano."

Partiendo de esta definición, se toma en cuenta cuál ha sido el impacto a nivel local, para tener una mejor visión de qué tan prevalente es en la población estudiada, de Manta. Según la información obtenida el 3 de septiembre por el Instituto Nacional de Investigación en Salud Pública (INSPI, 2020): "113.767 son el total de casos confirmados (...) Los casos en provincias son Galápagos 109, Guayas 18.836, 
Santo Domingo de los Tsáchilas 4.505, Esmeraldas 3.543, Manabí 8.482, Santa Elena 1.297, Los Ríos 3.273 y El Oro 4.385 (...) Pichincha 23.898." Como puede apreciarse la provincia de Manabí se encuentra en el tercer lugar de incidencia en los casos con COVID-19 a nivel de país, resultando una de las más afectadas.

Existe un sinnúmero de estudios nuevos en la actualidad que determinan que el estrés es un factor de importancia incluso a un nivel global, expresando que diferentes circunstancias presentes en la respuesta de la sociedad a los problemas globales generan diferentes posibles niveles de ansiedad. Este hecho tiene una particular expresión en el afrontamiento a la pandemia del coronavirus, tal como se expresa en los escritos de Boyraz y Legros (2020) donde se le correlaciona con el estrés postraumático:

COVID-19 continúa extendiéndose rápidamente por todo el mundo, afectando a más personas todos los días de varias maneras (por ejemplo, pérdidas financieras, pérdida de empleo, dificultad para acceder a suministros vitales, aumento del aislamiento social, incertidumbres sobre el futuro). (...) Si bien algunos países actualmente están menos afectados por COVID-19, los residentes de estos países aún pueden experimentar ansiedad anticipada debido a la rápida propagación del virus. La investigación sugiere que la ansiedad anticipatoria es una experiencia común durante $y$ después de brotes de enfermedades infecciosas. (...) En consecuencia, pueden estar en mayor riesgo de desarrollar trastorno de estrés postraumático y otros problemas de salud mental. Aunque los factores de riesgo para la salud mental asociados con COVID-19 aún no se han estudiado sistemáticamente. [Traducido del inglés por el autor].

Un buen indicio de que un acontecimiento se encuentra prevalente en su población es cuando existe un cambio conductual $y$ de sus costumbres a un nivel macro y en diferentes sociedades; no solo afectando a las personas que se encuentran diagnosticadas, sino a todas las personas de las comunidades donde viven. Vera (2020) refiere sobre la población de Paraguay que: "Un estudio 
cualitativo informó que varios participantes describieron cambios de comportamiento a largo plazo después del período de cuarentena, como el lavado de manos vigilante y evitar las multitudes y, para algunos, el regreso a la normalidad se retrasó."

El comportamiento de las poblaciones ante la pandemia, como ocurre ante eventos que implican modificación en sus hábitos y conductas, está mediado por su resiliencia. Esta constituye una categoría fundamental en este estudio. Sobre la importancia de la misma y su aplicabilidad, Uriarte (2005) nos dice lo siguiente: "Se trata de un dominio del conocimiento en el que confluyen observaciones (...) las cuales evidencian la capacidad del ser humano para resistir y superar las adversidades (...) a pesar de haber sufrido experiencias traumáticas."

Según la etología del término, Fores y Grane (2008) plantean que: "El termino Resiliencia proviene del latín Resilio que significa volver atrás, volver de un salto, rebotar, saltar hacia atrás, ser repelido o resurgir". Esto nos ofrece una idea simplificada de la aptitud de una persona con resiliencia, cuestión que está vinculada a otros estados mentales como son la ansiedad.

La resiliencia ha sido un punto de discusión en el campo humanístico, puesto que el término no proviene del mismo. Según su historia, Saavedra y Villalta (2008) nos expresan lo siguiente:

(...) tradicionalmente el término resiliencia se usa en el campo de la física y se refiere a la capacidad que tienen los cuerpos para volver a su forma original luego de haber sufrido deformaciones producto de la fuerza. Ciertamente al utilizar el término en ciencias sociales, debemos realizar la analogía con los cuerpos físicos y repensar el concepto en función de una resistencia del sujeto a las adversidades que le ofrece su ambiente. Para ello es necesario tener presente que, los seres humanos mostramos reacciones distintas, bajo estímulos similares. Lo mismo ocurre frente a estímulos dolorosos o situaciones de adversidad o estrés, en donde las reacciones de los sujetos serán variadas e incluso opuestas.

Se evidencia que la resiliencia es un concepto estrechamente 
relacionado con el estrés y por consecuencia con la ansiedad. La resiliencia es un factor protector para la ansiedad tal como lo plantea Márquez (2017) cuando se refiere a que las personas resilientes "Pueden reducir la intensidad del estrés, lo que hace que disminuya la presencia de signos emocionales negativos como la ansiedad y depresión al tiempo que aumenta la salud emocional."

Piña (2015) refiriéndose al concepto de resiliencia, comenta: "No se trata de un concepto cualquiera, por cuanto, y en principio, del mismo no podría siquiera decirse que tiene que ver con la fenomenología de lo psicológico, mucho menos que se distinga por su precisión y claridad."

Sería más beneficioso explicar la resiliencia por sus cualidades y no por una definición, la cual se asocia con el término de factor protector. En la prueba SV-RES se describen 4 ámbitos de profundidad de la resiliencia, cada uno corresponde a 3 factores de la resiliencia:

- Condiciones de base, corresponden a factores de: "identidad", son valores culturales que definen al sujeto; "vínculos", corresponde al valor de su círculo social primario; y "afectividad", describe la posibilidad de sí mismo y su relación con el entorno.

- Visión de sí mismo: "Autonomía", define su aporte particular al entorno; "redes", es el vínculo afectivo con su círculo social primario; "auto eficiencia", describe la posibilidad de éxito percibido.

- Visión del problema: "Satisfacción", cómo se interpreta la situación problema; "modelos", papel de la red social para superar problemáticas; "aprendizaje", valor percibido de aprendizaje de una problemática.

- Respuesta resiliente: "Pragmatismo", interpretación de acciones realizadas; "metas", valor del contexto de las metas y red social sobre una problemática; "generatividad", percepción de poder pedir ayuda para resolver una problemática.

Estos 12 factores son analizados en 60 ítems con afirmaciones de "yo soy", "yo estoy" y "yo tengo", utilizados para la medición de la resiliencia. Los mismos se describen como factores fluentes, puesto que 
la resiliencia es algo fluido y cambiante. (Saavedra y Villalta, 2008, p. 30-34).

Respecto a la validez y confiabilidad de la prueba mencionado para ser utilizado en este estudio, se considera a partir de la evaluación de su efectividad que: "(...) la escala de Resiliencia (SV-RES) (...) evidenció una apropiada y satisfactoria validez de estructura interna, validez convergente, así como una adecuada confiabilidad de consistencia interna de la escala en los puntajes obtenidos." (Janine, 2020).

Una de las variables de estudio es la ansiedad, presentada y descrita por Romero (2009) como la: "reacción global del organismo ante una situación que percibe como amenazante a su existencia y que incluye aspectos emocionales, cognitivos, somáticos y conductuales. La ansiedad es patológica por su forma de presentación, su intensidad, su persistencia y su recurrencia inmotivadas."

Esta se presenta por una aglomeración de síntomas que se pueden observar o describir en el paciente o personas estudiadas. Una medición bastante usual para este propósito es utilizar test psicométricos para obtener información válida al realizar un estudio 0 describir una sintomatología de un paciente. Entre las más usadas tenemos la prueba de ansiedad de Hamilton. Como lo indica Thompson (2015): "Es una de las primeras escalas de calificación para medir la gravedad de los síntomas de ansiedad percibidos. Todavía se usa en la actualidad, se considera una de las escalas de calificación más utilizadas." [Traducida del inglés por el autor].

Para una descripción de la estructura de la prueba, su propio creador Hamilton (1959) refiere lo siguiente: "La escala consta de 14 ítems, cada uno definido por una serie de síntomas, y mide tanto la ansiedad psíquica (agitación mental y angustia psicológica) como la ansiedad somática (quejas físicas relacionadas con la ansiedad)." [Traducido del inglés por el autor].

Este planteamiento conduce a una distinción importante, entre ansiedad como estado y la ansiedad como rasgo. Ambas describen la misma sintomatología, pero difieren en los 
momentos y circunstancias que se presentan. Entonces, según los escritos de Zayas, Gil-Olarte, Guerrero y Guil (2017) estas distinciones se expresan de la forma siguiente: "Ansiedad estado: condición emocional transitoria del organismo humano caracterizada por sentimientos subjetivos conscientemente percibidos, detención y aprensión. (...) Ansiedad rasgo: relativa propensión ansiosa por la que difieren los sujetos en su tendencia a percibir las situaciones como amenazadoras."

Cuando se aborda la relación entre la resiliencia y la ansiedad, se consideran ambos elementos, relacionados con la salud mental. Algunos estudios describen la influencia que tiene la resiliencia sobre la ansiedad, entre los que se encuentran los de Zayas, Gil-Olarte, Guerrero y Guil (2017) acerca de madres de menores que padecen de diabetes tipo 1. Al respecto se señala que: “(...) las madres que consiguen una visión más equilibrada de las circunstancias adversas y toman los acontecimientos con actitudes más positivas, podrían reducir los niveles de ansiedad estado".

Por último, existen estudios que han demostrado una relación entre ansiedad y resiliencia en diferentes circunstancias. Tal es el caso del desarrollado con una población de deportistas (Valdivia, 2016) en el que se comprueba que: "Existen diferencias entre la ansiedad estado y la resiliencia. Una ansiedad estado bajo proporciona mayores niveles de resiliencia (...) en una ansiedad rasgo alta, se tienen menores niveles de resiliencia y en una ansiedad rasgo baja se tienen mayores niveles de resiliencia."

En un entorno académico los resultados de la relación entre ansiedad y resiliencia según Silva, José, Astorga y Consuelo (2015) fueron los siguientes: "existe una relación negativa entre la resiliencia académica y la ansiedad. Los alumnos que son competentes para resolver los contratiempos propios de los deberes académicos son menos ansiosos." Se observa que incluso, en diferentes áreas y poblaciones, existe una estrecha relación entre los factores mencionados.

Otro estudio que corrobora lo anteriormente descrito, que considera la ansiedad como un trastorno psicopatológico; es el de 
Fernandes, Teva y de la Paz (2015) donde se plantea: "Respecto a las categorías temáticas, existe una asociación entre los altos niveles de resiliencia y los bajos niveles de trastornos psicopatológicos."

La influencia favorable de la resiliencia sobre la ansiedad se puede observar en el estudio de Álvarez (2018): "La resiliencia es un factor protector que minimiza la ocurrencia de síntomas globales del Trastorno de Ansiedad Generalizada. Igualmente, es un factor protector en la medida en que reduce la probabilidad de riesgo frente a episodios comórbidos a esta enfermedad."

Los estudios referenciados dan cuenta de la relación entre resiliencia y ansiedad en diferentes poblaciones. A partir de estas experiencias previas, se propone la búsqueda de la relación entre resiliencia y ansiedad en pacientes con COVID-19. Por lo tanto, se plantea como propósito del estudio el determinar los niveles de resiliencia $y$ ansiedad en las personas infectadas por COVID-19, así como la relación entre estas categorías.

\section{Metodología (Materiales y métodos)}

Se realizó una investigación de tipo descriptiva con un enfoque cualicuantitativo para profundizar en las categorías estudiadas. El método utilizado es el estudio de caso, el cual permite la exploración intensiva de las categorías seleccionadas en la investigación.

Su finalidad se asocia a captar la esencia de la expresión de la resiliencia y la ansiedad en la muestra estudiada, es decir, comprender sus particularidades. Se aplicaron además los métodos inductivo-deductivo, análisissíntesis-comparación.

Como instrumentos se emplearon el Test SV-RES sobre Resiliencia (Saavedra \& Villalta, 2008) compuesto por 60 ítems, que se corresponden con 12 dimensiones de la resiliencia.

Adicionalmente se aplicó el Test de Ansiedad de Hamilton (HAM-A) para determinar el nivel de ansiedad, compuesto por 14 ítems, que valoran sintomatología somática y psíquica de ansiedad.

Se desarrolló una entrevista abierta 
para obtener información relevante respecto a las categorías investigadas. Por su carácter abierto, la entrevista, permite la expresión integral del sujeto a partir de las preguntas sugeridas, en estrecho vínculo con la problemática investigada, además de complementar los datos obtenidos en el resto de los instrumentos empleados en la investigación.

El acceso al campo se produjo a través de herramientas virtuales, tales como: computadoras y celulares con acceso a internet.

Como población se toman las personas que fueron diagnosticadas de COVID-19 sin distinción de edad o sexo, durante la pandemia del 2020 en la ciudad de Manta, Manabí, Ecuador, entre los meses de junio y julio. La muestra quedó conformada por 5 personas; a quienes, dada la complejidad de la situación de aislamiento, se entrevistaron por medio de telecomunicaciones. Las mismas fueron seleccionadas con carácter intencional, asumiendo como criterios de inclusión que hayan tenido un diagnóstico positivo de COVID-19, teniendo sintomatología de bajo riesgo y que estuvieran en una recuperación reciente (de 3 a 6 semanas).

\section{Análisis e interpretación de resultados}

Para procesar la información obtenida se presentan los hallazgos de la investigación resultado de la aplicación de los instrumentos "Escala de Resiliencia SV- RES", "Escala de ansiedad de Hamilton" y las respuestas dada por los sujetos a la entrevista abierta que complementa las informaciones obtenidas en estos instrumentos.

En primer lugar, se exponen los resultados generales obtenidos en la categoría resiliencia, a través del comportamiento de los ámbitos de profundidad en los que se evalúa la categoría. Posteriormente se describen los resultados en cuanto a la categoría ansiedad, especificando la ansiedad psíquica y la somática.

\subsection{Figuras y Tablas de los resultados obtenidos en la categoría resiliencia, así como en sus ámbitos de profundidad.}

La categoría resiliencia evaluada a través de los puntajes percentiles del Test SV-RES determinan el rango 
categorial de las personas. Entre $0 \mathrm{y}$ 25 es considerado con resiliencia de nivel "Bajo" de 26 a 74 se considera "Promedio" y de 75 a 99 determina que es "alto".

Figura 1. Niveles de Resiliencia de las personas diagnosticadas con COVID-19.

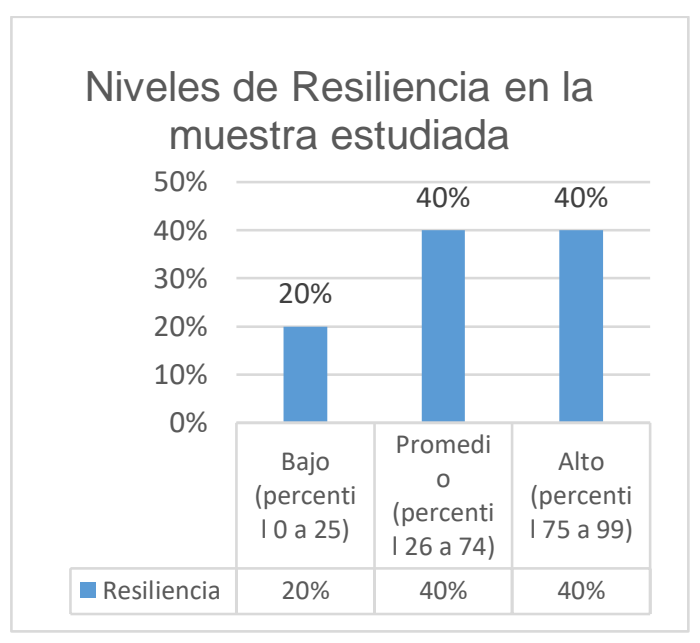

Fuente: Tirino y Giniebra (2020).

De acuerdo con la figura 1, se puede determinar que el $40 \%$, equivalente a 2 personas, responden que su nivel de resiliencia es elevado, al igual que los que presentan similar percentil un nivel promedio, mientras que el siguiente $20 \%$, equivalente a 1 persona, su nivel de resiliencia es bajo.

Al explorar los ámbitos de profundidad, el primero de ellos, referido a las condiciones de base, se parte de que son un sistema de creencias y vínculos sociales que guían acciones expresadas en los factores de identidad, vínculos y afectividad. El comportamiento de estos factores es el siguiente:

Figura 2. Niveles del Factor Identidad en las personas diagnosticadas con COVID-19.

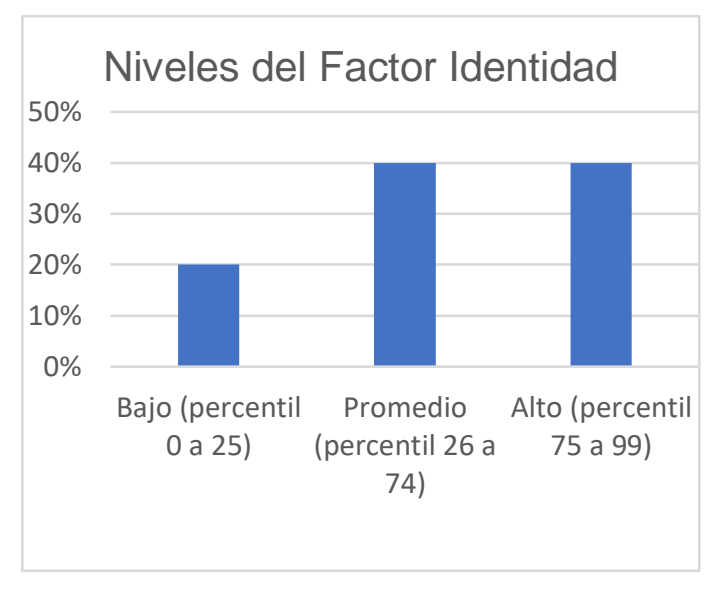

Fuente: Tirino y Giniebra (2020).

De acuerdo con la figura 2, se puede determinar que el $40 \%$, equivalente a 2 personas responden que su nivel de identidad es elevado, al igual que los que presentan un nivel promedio con igual percentil, mientras que el siguiente $20 \%$, equivalente a 1 persona, presenta un nivel de identidad bajo.

Figura 3. Niveles del Factor Vínculos de las personas diagnosticadas con COVID-19.

\section{Niveles del Factor Vínculos}

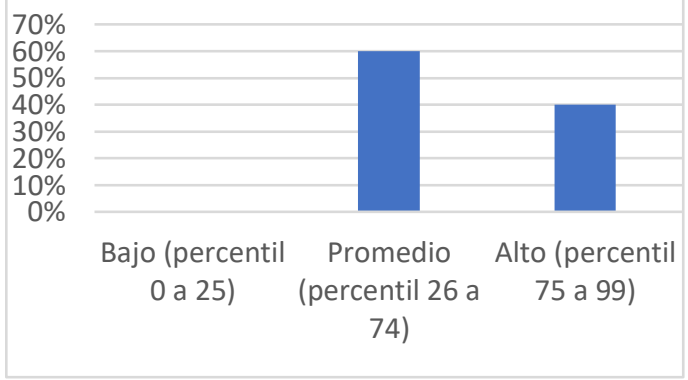

Fuente: Tirino y Giniebra (2020). 
De acuerdo con la figura 3, se puede determinar que el $60 \%$, equivalente a 3 personas, presenta un nivel de vínculos promedio, mientras que el $40 \%$, equivalente a 2 personas, responden con un nivel de vínculos elevado. Ningún sujeto presenta nivel de vínculos bajo.

Figura 4. Niveles del Factor Afectividad de las personas diagnosticadas con COVID-19.

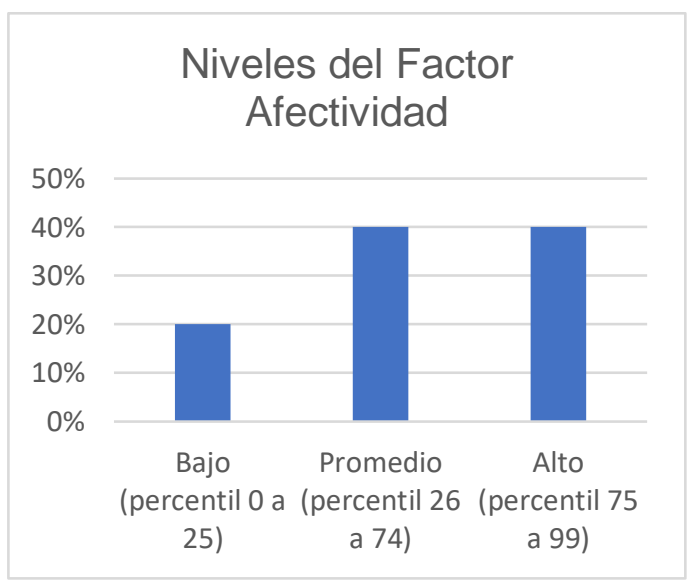

Fuente: Tirino y Giniebra (2020).

De acuerdo con la figura 4, se puede determinar que el $40 \%$, equivalente a 2 personas responden que su nivel de afectividad es elevado, al igual que los que presentan en igual percentil un nivel promedio. El 20\%, equivalente a 1 persona, presenta un nivel de afectividad bajo.

En este grupo de categorías referidas a las condiciones de base, se ha de destacar el factor vínculos puesto que corresponde al valor del círculo social primario, que muestra los niveles más altos y no se obtiene ningún puntaje de resiliencia bajo. En las entrevistas abiertas, se corrobora esta información pues los participantes denotan la importancia de sus vínculos sociales para su salud mental, tales como su familia o su conyugue.

En el segundo ámbito de profundidad, referido a la visión de sí mismo, este corresponde a elementos afectivos y cognitivos positivos 0 proactivos ante los problemas; apegados a un contexto e historia, que contemplan factores de autonomía, redes y autosuficiencia.

Figura 5. Niveles del Factor Autonomía de las personas diagnosticadas con COVID-19.

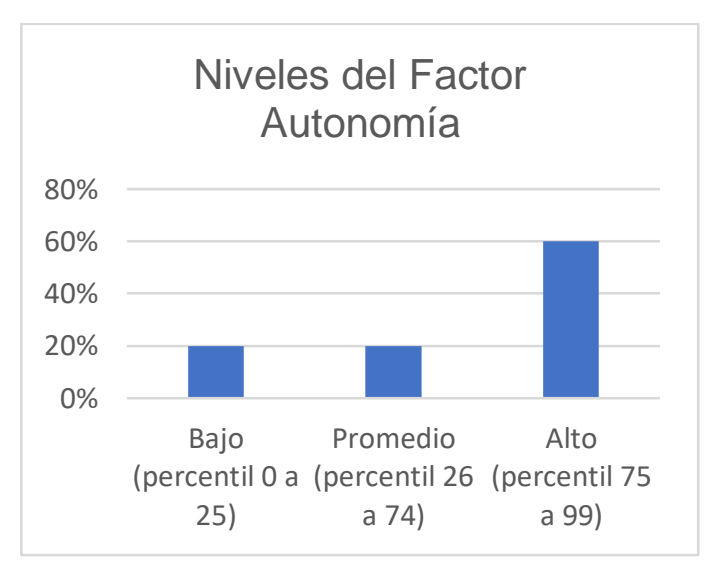

Fuente: Tirino y Giniebra (2020).

De acuerdo con la figura 5 , se puede determinar que en el $60 \%$, equivalente a 3 personas, el nivel de modelos es elevado. El 20\%, 
equivalente a 1 persona responde que su nivel de modelos es promedio, al igual se presenta en igual percentil un nivel bajo.

Figura 6. Niveles del Factor Redes de las personas diagnosticadas con COVID-19.

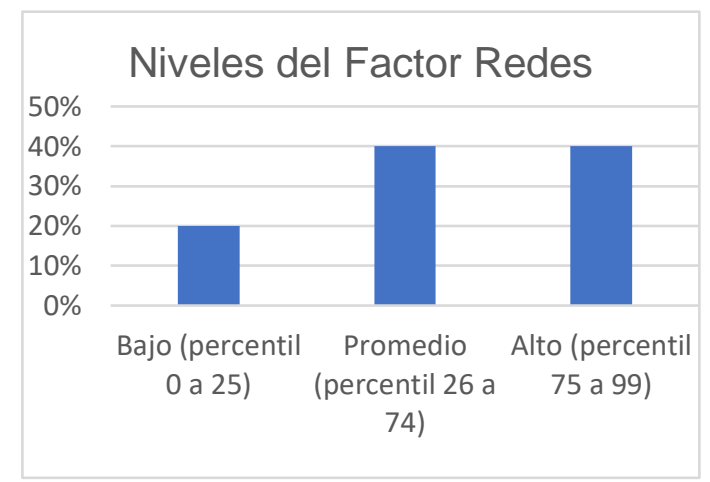

Fuente: Tirino y Giniebra (2020).

De acuerdo con la figura 6 , se puede determinar que el $40 \%$, equivalente a 2 personas, responden que su nivel de redes es elevado, al igual que los que presentan en igual percentil un nivel promedio, mientras que el siguiente $20 \%$, equivalente a 1 persona, su nivel de redes es bajo.

Figura 7. Niveles del Factor Autosuficiencia de las personas diagnosticadas con COVID-19.

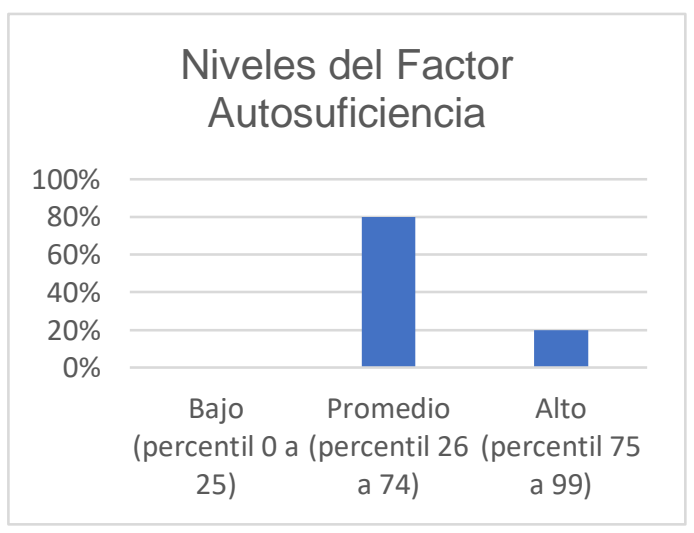

Fuente: Tirino y Giniebra (2020).
De acuerdo con la figura 7 , se puede determinar que el $80 \%$, equivalente a 4 personas, presentan un nivel de autosuficiencia promedio. El 20\%, equivalente a 1 persona responde que su nivel de autosuficiencia es elevado. Ningún sujeto presenta nivel de autosuficiencia bajo.

En este ámbito de la visión de sí mismo, se quiere denotar el factor de autosuficiencia, el cual describe la posibilidad de éxito percibido ante la problemática por la que atraviesan los sujetos. Este factor resulta el más sobresaliente en cuanto a los puntajes obtenidos dentro de este ámbito de profundidad en la medición la resiliencia, no contiene ningún puntaje de resiliencia bajo.

En las entrevistas, los sujetos expresaron en su mayoría, que después de la sintomatología tenían una buena percepción del manejo de la problemática. Dentro de este ámbito, le sigue como significativo, la autonomía, que define el aporte particular del sujeto al entorno.

Este factor obtiene el mayor porcentaje en resiliencia alta. En las entrevistas abiertas denotan que después de la sintomatología, tenían una mejor visión de aporte para sus 
familias puesto que podían trabajar y ayudar en la situación económica familiar.

El tercer ámbito de profundidad para caracterizar la resiliencia lo constituye la visión del problema; denota qué tan abordable es la problemática y su conducta recurrente. Se contemplan factores de satisfacción, modelos y aprendizaje.

Figura 8. Niveles del Factor Satisfacción de las personas diagnosticadas con COVID-19

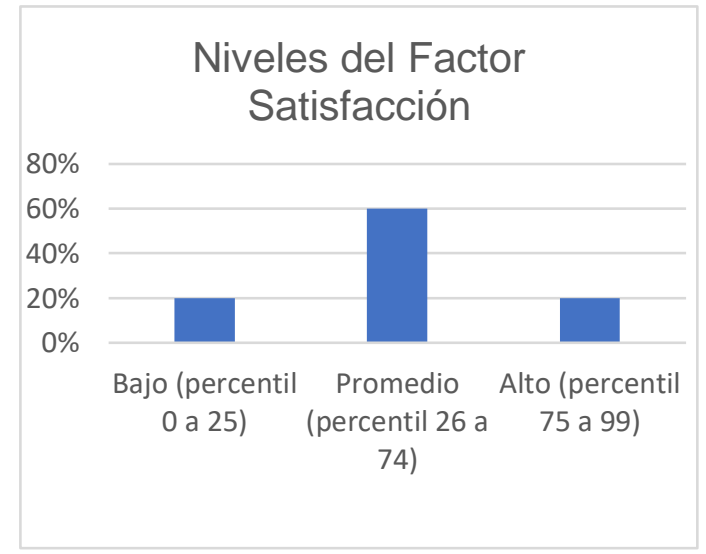

Fuente: Tirino y Giniebra (2020).

De acuerdo con la figura 8, se puede determinar que el siguiente $60 \%$, equivalente a 3 personas, presentan un nivel de satisfacción promedio, mientras que el $20 \%$, equivalente a 1 persona responde que su nivel de satisfacción es elevado, al igual que los que presentan un igual percentil en nivel bajo.
Figura 9. Niveles del Factor Modelos de las personas diagnosticadas con COVID-19.

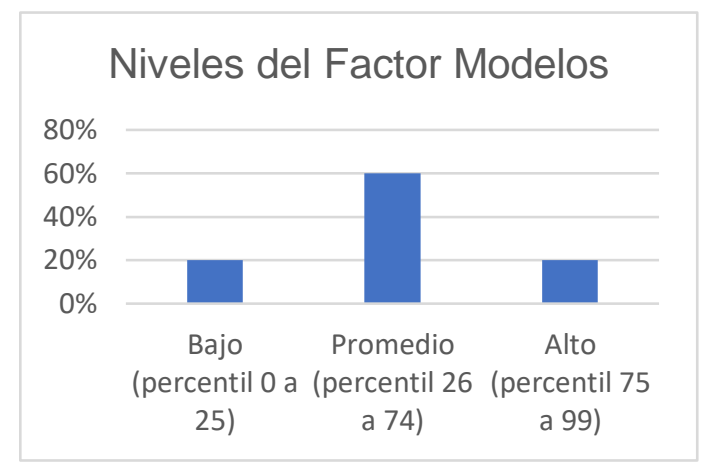

Fuente: Tirino y Giniebra (2020).

De acuerdo con la figura 9, se puede determinar que en el $60 \%$, equivalente a 3 personas, el nivel de modelos es promedio. El 20\%, equivalente a 1 persona responden que su nivel de modelos es elevado, al igual que los que presentan en igual percentil un nivel bajo.

Figura 10. Niveles del Factor Aprendizaje de las personas diagnosticadas con COVID-19.

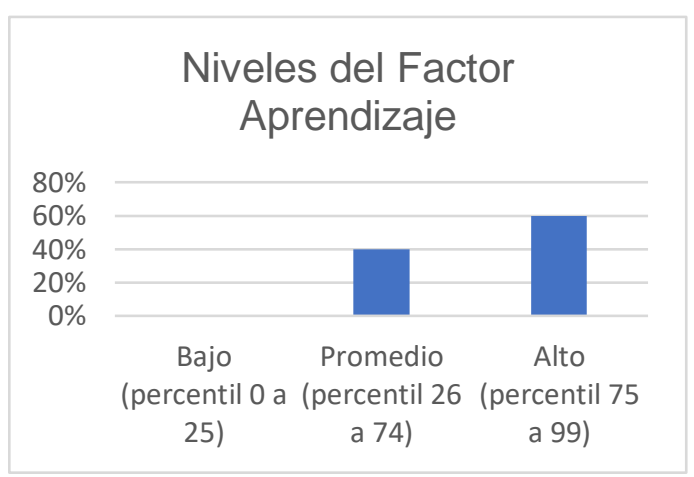

Fuente: Tirino y Giniebra (2020).

De acuerdo con la figura 10, se puede determinar que el 60\%, equivalente a 3 personas responden que su nivel de aprendizaje es elevado, mientras que el siguiente 
$40 \%$, equivalente a 2 personas, presentan un nivel de aprendizaje promedio. Ningún sujeto presenta nivel de aprendizaje bajo.

En este grupo de factores concernientes a la visión del problema, se denota el factor de aprendizaje, el cual describe valor percibido de aprendizaje de una problemática, como el más significativo, obteniendo los mayores puntajes y no presentando ningún puntaje de resiliencia bajo.

El último ámbito de profundidad referido a la Respuesta Resiliente, esta es una acción orientada a metas, respuestas sustentadas 0 vinculadas a la visión de la problemática. Contiene tres factores de pragmatismo, metas $y$ generatividad.

Figura 11. Niveles del Factor Pragmatismo de las personas diagnosticadas con COVID-19.

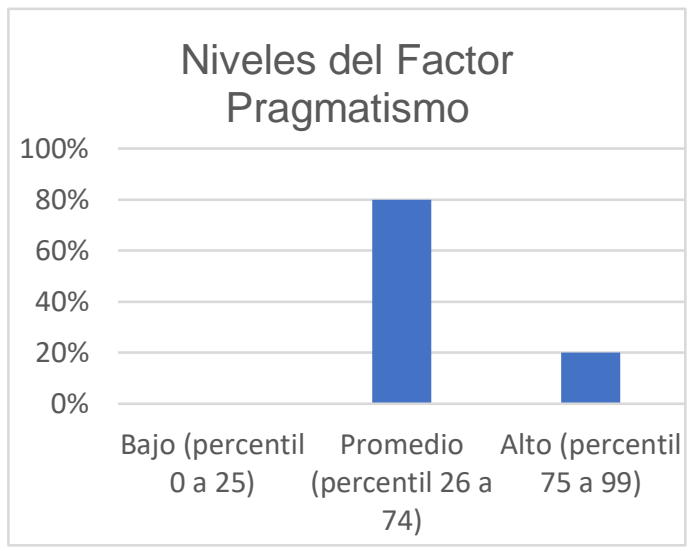

De acuerdo con la figura 11, se puede determinar que el siguiente $80 \%$, equivalente a 4 personas, presentan un nivel de pragmatismo promedio. El $20 \%$, equivalente a 1 persona, responde que su nivel de pragmatismo es elevado. Ningún sujeto ofreció respuesta con nivel de pragmatismo bajo.

Figura 12. Niveles de Factor Metas de las personas diagnosticadas con COVID-19.

\section{Niveles del Factor Metas}

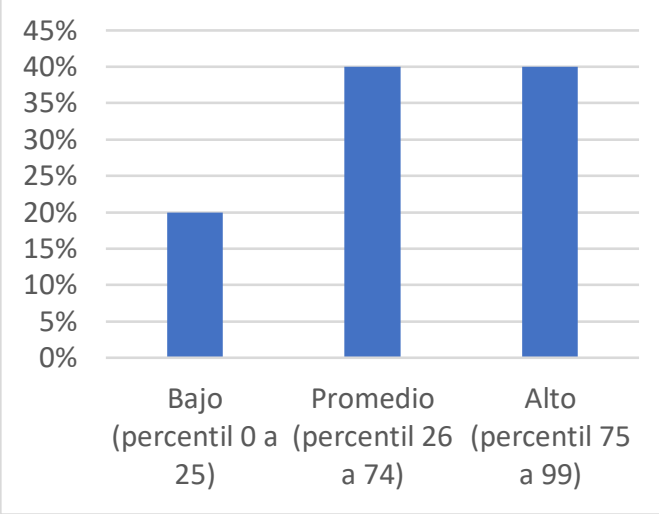

Fuente: Tirino y Giniebra (2020).

En cuanto al factor metas, dentro del ámbito respuesta Resiliente, en la figura 12 , se puede determinar que el $40 \%$, equivalente a 2 personas responden que su nivel de metas es elevado, al igual que los que presentan un igual percentil en nivel promedio, mientras que el siguiente $20 \%$, equivalente a 1 persona, su nivel de metas es bajo.

Fuente: Tirino y Giniebra (2020). 
Figura 13. Niveles de Factor Generatividad de las personas diagnosticadas con COVID-19.

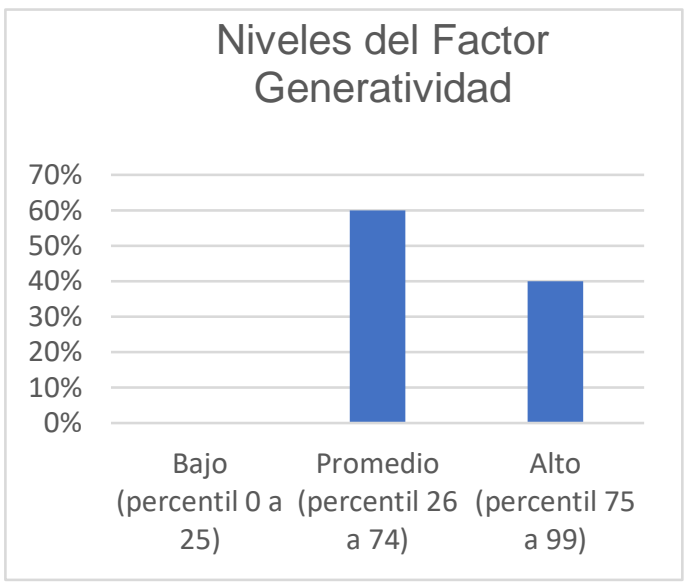

Fuente: Tirino y Giniebra (2020).

En cuanto al factor generatividad, de acuerdo con la figura 13, se puede determinar que el $60 \%$, equivalente a 3 personas, presentan un nivel de generatividad promedio, mientras que el $40 \%$, equivalente a 2 personas responden que su nivel de generatividad es elevado. Ningún sujeto presenta nivel de generatividad bajo.

En este grupo de factores relacionados con el ámbito de respuesta Resiliente resulta significativo el factor de generatividad, el cual describe se relaciona con la percepción de poder pedir ayuda para resolver una problemática. Este factor obtiene los valores más significativos y no contiene ningún puntaje de resiliencia bajo, al igual que el factor pragmatismo vinculado a la interpretación de las acciones realizadas para asumir la problemática porque las que atraviesan los sujetos.

\subsection{Figuras y Tablas de los resultados obtenidos en la categoría ansiedad: la ansiedad psíquica y la somática.}

En cuanto a la categoría ansiedad se evalúa a través de un rango de puntajes dados por la escala del Test de Ansiedad de Hamilton (HAM-A). Los rangos prevén que con menos de 17 se considera con estado de ansiedad "Bajo" de 18 a 24 se considera "Moderado" y puntajes superiores 0 iguales a 25, determinan que es "Severo".

Figura 14. Niveles de Ansiedad de las personas diagnosticadas con COVID-19.

\section{Niveles de Ansiedad en la muestra estudiada}

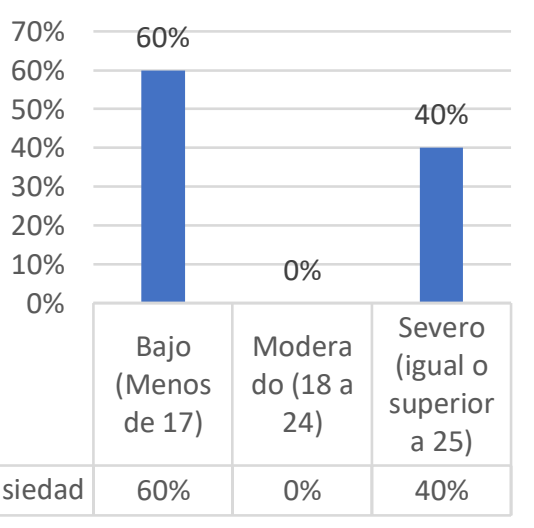

Fuente: Tirino y Giniebra (2020). 
De acuerdo con la figura 14, se puede determinar que el $60 \%$ equivalente a 3 personas, presentan un nivel de ansiedad bajo, le sigue el $40 \%$, que equivale a 2 personas con nivel de ansiedad severo. No existe ningún sujeto con nivel de ansiedad moderado.

Para la calificación del este test se sigue la referencia del Centro Nacional de Excelencia Tecnológica en Salud de México (CENETEC, 2010), donde se cita a Sánchez y López (2005) quienes mencionan la consideración de ansiedad somática y ansiedad psíquica:

Tanto para considerar la ansiedad somática como la psíquica, se consideran los rangos siguientes: "Ansiedad Ausente (Puntuaciones entre 0 a 5). Ansiedad Leve (Puntuaciones entre 6 a 14). Ansiedad Moderado a Grave (Puntuaciones Mayor o igual a 15)".

En los gráficos siguientes se muestran niveles de ansiedad psíquica (agitación mental y angustia psicológica) como la ansiedad somática (quejas físicas relacionadas con la ansiedad).
Figura 15. Niveles de Ansiedad psíquica de las personas diagnosticadas con COVID-19.

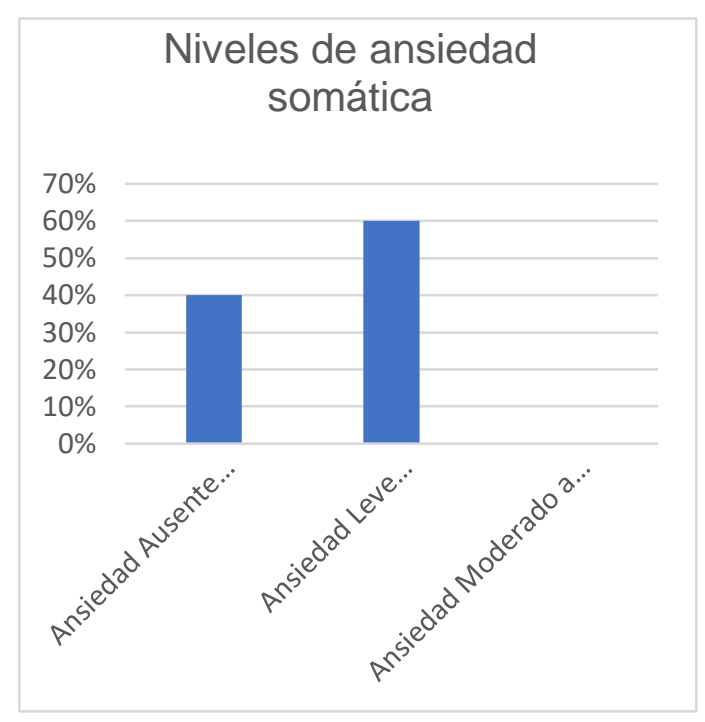

Fuente: Tirino y Giniebra (2020).

De acuerdo con la figura 15, se puede determinar que el $40 \%$ equivalente a 2 personas, presentan un nivel de ansiedad psíquica ausente, al igual que ansiedad moderado a grave, mientras que el $20 \%$ corresponde a una ansiedad leve.

Figura 16. Niveles de Ansiedad somática de las personas diagnosticadas con COVID-19.

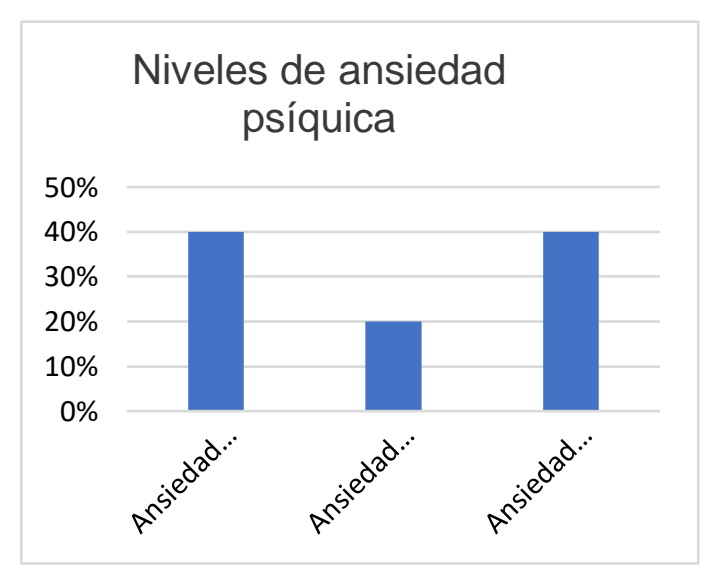

Fuente: Tirino y Giniebra (2020). 
De acuerdo con la figura 16, se puede determinar que el $60 \%$ corresponde a una ansiedad leve, mientras que el $40 \%$ equivalente a 2 personas, presentan un nivel de ansiedad somática ausente. No se detecta ningún sujeto que presente un nivel alto de ansiedad somática.

Se denota que la población obtiene los mayores puntajes en la ansiedad psíquica y no se obtiene ningún puntaje en ansiedad somática de nivel moderado a grave. Lo anterior concuerda con la información obtenida en la entrevista abierta en la cual el total de la muestra describió un estado ansioso durante la sintomatología, pero sólo un porcentaje ínfimo en cambios físicos como de sueño o de alimentación que corresponden a síntomas de ansiedad somática.

\section{Conclusiones}

Se estudiaron los niveles de ansiedad y de resiliencia de la población de personas que fueron diagnosticadas con COVID-19 en la ciudad de Manta, Manabí, Ecuador, entre los meses de junio y julio de 2020.

Se pudo determinar que existe una relación entre la ansiedad y la resiliencia en la muestra estudiada, esto concuerda con investigaciones ejecutadas por Valdivia (2016), Silva, José, Astorga y Consuelo (2015) y Fernandes, Teva y de la Paz (2015), las cuales plantean una relación significativa entre estas categorías.

Un reciente estudio de Gennaro, M., De Lorenzo, R., y Poletti, S. (2020) presentan que: "Los sobrevivientes de COVID-19 presentaron una alta prevalencia de secuelas psiquiátricas emergentes, y el $55 \%$ de la muestra presentó una puntuación patológica para al menos un trastorno. (...) se espera una incidencia de (..) ansiedades mayores que el promedio.".

Esto indica la necesidad de tomar medidas preventivas para evitar el desarrollo de patologías. Entre ellas podría considerarse el aumento de la resiliencia y las herramientas para el manejo de la ansiedad.

Ehmke (2020), citando a Bubrick, refiriéndose a los tratamientos para la ansiedad considera necesario usar la tolerancia contra la incertidumbre (2020). Este elemento genera cierta inmunidad psicológica, por cuanto prepara al sujeto para 
lidiar con situaciones imprevistas y amenazantes como lo constituye el diagnóstico y evolución de esta enfermedad.

Al analizar los datos obtenidos en las categorías estudiadas, de manera individual, se constata lo siguiente: las personas que obtuvieron puntajes en niveles altos de resiliencia presentaron bajos niveles de ansiedad y un solo sujeto de la muestra obtuvo un nivel de resiliencia bajo con un nivel de ansiedad severo.

Con respecto a los ámbitos de profundidad de la resiliencia, se corrobora en la entrevista abierta, que los factores de protección más relevantes son: vínculos, autosuficiencia, aprendizaje, pragmatismo y generatividad.

El presente estudio tuvo algunas limitaciones, tales como las siguientes: la muestra limitada de la población derivada de la dificultad para acceder a la misma por estar los sujetos en fase de recuperación, la restricción para el contacto directo impuesta por las condiciones de cuarentena, las medidas de seguridad tomadas para la protección de los participantes y la carencia de investigaciones previas suficientes sobre problemáticas psicológicas asociadas al COVID19, lo cual no permite acceso a fuentes y evidencias amplias por cuanto es un tema de reciente exploración científica.

Se recomienda en futuras investigaciones, extender a mayor número de personas los estudios, para desde un enfoque cuantitativo, obtener tendencias o regularidades en el comportamiento de estas categorías en muestras más amplias de la población.

Los resultados obtenidos en la muestra estudiada permiten plantear las siguientes conclusiones: Los niveles de resiliencia en la muestra se comportan entre promedio y alto, resultando caracterizados por una percepción significativa del círculo social primario, la existencia de habilidades y de una percepción favorable de resolución del problema.

En cuanto a los niveles de ansiedad, esta se manifiesta en un nivel bajo, predominando la ansiedad psíquica. Esto indicaría que existe una mayor afectación emocional y cognitiva y no en los aspectos fisiológicos 
asociados a la ansiedad.

Prácticamente no aparecen registros acerca de la ansiedad somática que se presentan cuando existen serias afectaciones en la salud mental producto de la ansiedad.

En cuanto a la relación entre las categorías resiliencia y ansiedad, se observa que los niveles de resiliencia presentes permiten a los sujetos el control de la ansiedad, como un factor protector, la cual se manifiesta en niveles bajos.

A su vez, el control de la ansiedad permite sostener un nivel resiliencia adecuado para el afrontamiento a la problemática por la que atraviesan los sujetos, estableciéndose como una co-determinación dialéctica entre estas categorías de estudio en la muestra.

\section{Bibliografía}

Álvarez, D. (2018). La resiliencia como factor protector en sujetos con trastorno de ansiedad generalizada. Recuperado de: https://repository.usc.edu.co/ bitstream/20.500.12421/159/ 1/LA\%20RESILIENCIA\%20C OMO.pdf

Boyraz, G. y Legros, D. (2020) Coronavirus Disease (COVID-
19) and Traumatic Stress: Probable Risk Factors and Correlates of Posttraumatic Stress Disorder.

CENETEC. (2010). Diagnóstico y tratamiento de los trastornos de ansiedad en el adulto. Recuperado de: http://www.cenetec.salud.gob .mx/descargas/gpc/Catalogo Maestro/392_IMSS_10_Ansie dad/EyR_IMSS_392_10.pdf

Ehmke, R. (2020). Lidiar con la ansiedad y el coronavirus. Recuperado de: https://childmind.org/article/lid iar-con-la-ansiedad-y-elcoronavirus/

Fernandes, L. (2015). Resiliencia en adultos: una revisión teórica. Recuperado de: https://scielo.conicyt.cl/scielo. php?pid=s071848082015000300009\&script $=$ sci_arttext\&tlng=en

Fínez, J. y Morán, C. (2015). La resiliencia y su relación con salud y ansiedad en estudiantes españoles. International Journal of Developmental and Educational Psychology, vol. 1, núm. 1, pp. 409-416 Asociación Nacional de Psicología Evolutiva y Educativa de la Infancia, Adolescencia y Mayores Badajoz, España. Recuperado de: https://www.redalyc.org/pdf/3 498/349851779041.pdf 
Fores, A. (2008) La resiliencia. Recuperado de: https://books.google.es/books $? \mathrm{hl}=\mathrm{es} \& \mathrm{l} \mathrm{r}=\& \mathrm{id}=0 \mathrm{vOkDwAAQ}$ $B A J \& o i=f n d \& p g=P T 6 \& d q=r e s$ iliencia\&ots=vdme3SNAHM\& sig=S6NAILrjZ67oKmTnX0gx oKqEbhw\#v=onepage\&q=resi liencia\& $f=f a l s e$

García, A., Gil-Olarte, P., Guerrero, C., y Guil, R. (2017) Resiliencia y ansiedad en madres de menores que padecen diabetes tipo 1 . Recuperado de: http://www.infad.eu/RevistalN FAD/OJS/index.php/IJODAE P/article/view/982/864

Gennaro, M., De Lorenzo, R., y Poletti, S. (2020). Ansiedad y depresión en los sobrevivientes de COVID-19. Recuperado de: https://www.intramed.net/cont enidover .asp? contenidoid $=96$ 529

Hamilton M. (1959) Hamilton Anxiety Rating Scale (HAM-A). Recuperado de: https://dcf.psychiatry.ufl.edu/fi les/2011/05/HAMILTON-

ANXIETY.pdf

INSPI. (2020). Actualización de casos de coronavirus en Ecuador. Recuperado de: https://www.salud.gob.ec/actu alizacion-de-casos-decoronavirus-en-ecuador/

Janine, S. (2020). Propiedades psicométricas de la escala de resiliencia SV-RES para adultos mayores. Recuperado de:

https://repositorioacademico. upc.edu.pe/bitstream/handle/ 10757/625077/Moscoso_ES. pdf? sequence $=5 \&$ isAllowed $=$ $y$

Márquez, M. (2017) La resiliencia como factor protector de salud mental en adolescentes de una unidad educativa de valencia, estado Carabobo Recuperado de: http://mriuc.bc.uc.edu.ve/bitst ream/handle/123456789/574 $5 /$ mmarquez.pdf?sequence $=1$ \#: :text=La\%20resiliencia\%2 0es\%20un\%20factor,la\%20a daptaci\%C3\%B3n\%20en\%20 la\%20adultez.

ONU. (2020). Coronavirus. Recuperado de: https://www.who.int/es/healthtopics/coronavirus

Piña, J. (2015). Un análisis crítico del concepto de resiliencia en psicología. Recuperado de: https://revistas.um.es/analesp s/article/view/analesps.31.3.1 $85631 / 180381$

Romero, J. (2009). Prevalencia de ansiedad y depresión en pacientes con $\mathrm{VIH} /+$. Otras enfermedades infectocontagiosas. Revista Neurología, Neurocirugía y Psiquiatría; 42(1-4): Ene.-Dic: 25-31 Recuperado de: https://www.medigraphic.com /pdfs/revneuneupsi/nnp- 


\section{9/nnp091-4e.pdf}

Saavedra, E. y Villalta, M. (2008).

Escala de resiliencias sv-res

para jóvenes y adultos.

Recuperado de:

https://www.academia.edu/26

343874/Saavedra_-

Villalta_SV-

RES60_ESCALA_DE_RESIL

IENCIA_SV-RESn (páginas 30 - 34)

Thompson, E. (2015) Hamilton Rating Scale for Anxiety (HAM-A). Recuperado de: https://academic.oup.com/occ med/article/65/7/601/1733495

Uriarte, J. (2005). La resiliencia. Una nueva perspectiva en psicopatología del desarrollo. Recuperado de: https://www.redalyc.org/pdf/1 75/17510206.pdf

Vera, C. (2020) Cuarentena por COVID19 y Salud Mental. 